\title{
Association of Relative Handgrip Strength and Metabolic Syndrome in Korean Older Adults: Korea National Health and Nutrition Examination Survey VII-1
}

\author{
Seungyoun Hong* \\ Department of Senior Industry, Kangnam University, Yongin, Korea
}

Background: The prevalence of metabolic syndrome (MetS) is rapidly increasing with advanced age. Since aging affects sarcopenia and muscle strength, which could, in turns, affect MetS, it is critical to examine the association between MetS and muscle strength in this population. Handgrip strength (HGS) has been introduced as a simple assessment tool for muscular strength. Several studies investigating the association between HGS and the risk of MetS have yield conflicting results. Therefore, this study aimed to investigate the association of MetS and relative handgrip strength (RHGS) in elderly Koreans.

Methods: A total of 1,244 (male, 589; female, 655) elderly subjects (age $\geq 65$ years), who participated in the Seventh Korea National Health and Nutrition Examination Survey VII-1 (2016) were analyzed. Participant's HGS, body mass index (BMI), MetS, and its five criteria were measured. Subjects were categorized into four groups based on their RHGS, which was defined as absolute HGS divided by BMI.

Results: The highest quartile RHGS group has a significantly lower prevalence of MetS in both men and women ( $F=35.16, P<0.001$ vs. $F=31.78, P<0.001$, respectively). Moreover, the odds ratio of MetS was 0.52 times lower $(0.52 ; 95 \%$ confidence interval [Cl], 0.31-0.85) in the third quartile RHGS than that of the lowest RHGS, and 0.28 times lower $(0.28 ; 95 \% \mathrm{Cl}, 0.17-0.47)$ in the highest RHGS group in women than that of the lowest RHGS group. Conclusion: Maintaining a high level of HGS may reduce the incidence rate of MetS in elderly Koreans.
Received February 7, 2019

Reviewed February 28, 2019

Accepted March 10, 2019

${ }^{*}$ Corresponding author

Seungyoun Hong

(iD https://orcid.org/0000-0002-1574-9298

Department of Senior Industry, Kangnam University, 40 Gangnam-ro, Giheung-gu, Yongin 16979, Korea

Tel: +82-31-899-7210

Fax: +82-31-899-7211

E-mail:yoni91@hotmail.com

Key words: Hand strength, Metabolic syndrome, Aged, Muscle strength

\section{INTRODUCTION}

Metabolic syndrome (MetS) is a cluster of unhealthy cardiometabolic disease risk factors, such as abdominal obesity, high level of systemic hypertension, elevated fasting plasma glucose (FPG) level, high levels of triglyceride (TG), low levels of high-density lipoprotein cholesterol (HDL-C) defined by the American Heart Association. ${ }^{1}$ The prevalence of MetS is rapidly increasing with advanced age worldwide ${ }^{2-4}: 40 \%$ of people over 60 years in the US ${ }^{5}$ and $34.0 \%$ and $40.5 \%$ in elderly men and women in Korea. ${ }^{6}$ Since MetS is also known as being related to all-cause of mortality ${ }^{7}$, type 2 diabetes mellitus ${ }^{8}$, increased risk of cardiovascular disease (CVD), increased risk of cognitive decline ${ }^{9}$, and physical function, developing proper public health strategies for the detection, and prevention of MetS should be an urgent priority.

Further, population aging affects aging sarcopenia characterized by loss of muscle mass and muscle strength. ${ }^{10}$ It involves changes to underlying structures and processes, including muscle fibers and their 
innervation. Reduced muscle strength, in turn, can be used as a determinant for loss of physical function and disability risk in old age.

Handgrip strength (HGS) has been introduced as a simple and inexpensive assessment tool for muscular strength. Since HGS was strongly correlated with total muscle strength ${ }^{11}$, it is often used as an indicator of overall muscle strength for aging adults.

Numerous clinical and epidemiological studies show that low HGS is associated with a variety of poor health outcomes including subsequent onset of functional limitation ${ }^{10}$, chronic mobilities and disabilities $^{12}$, all-cause mortality ${ }^{13}$, and even longer length of stay among surgical patients. ${ }^{14}$

Several studies investigating the association between HGS and the risk of cardiovascular risk and MetS have yield conflicting results. Meta-analysis of cohort studies had reported that the hazard ratio was 1.6 for CVD when it compared the lowest versus the highest level of HGS. ${ }^{15}$ However, Gubelmann et al. ${ }^{16}$ reported no association between absolute HGS and CVD among 2,707 subjects over 50 years of age.

Besides, unclear sex difference in this association has been reported as well. Taiwanese study ${ }^{17}$ reported that reduced relative handgrip strength (RHGS) was associated with an increased cardiovascular risk score only in men and a recent UK study reported that HGS was associated with CVD only in men, not it women. ${ }^{18}$

Little is known concerning the relationship between MetS and HGS in the older adult who is a part of the population that has a higher incidence of MetS, activity of daily life (ADL) impairments and associated CVD than younger adults. During later life, the elderly are vulnerable to the adverse consequence of decreased HGS, i.e., frailty, disabilities, which required the long-term care. Many ADLs and instrumental activities of daily living such as eating, dressing, carrying groceries are involved in HGS. Those below 65 years of age living with MetS and decreased HGS may not need long-term care in the way that older people over 65 years of age would be due to the remained other physical function.

To our knowledge, studies of the association between HGS and MetS in the older population have not been reported in Korea. The increasing number of aging people with sarcopenia and an increase need of long-term care services demonstrate the need to conduct research identifying the association of HGS and risk factors. Therefore, the purpose of the current study was to investigate the associa-

tion of RHGS and MetS by analyzing the Korea National Health and Nutrition Examination Survey (KNHANES) VII-1, 2016.

\section{METHODS}

\section{Study subjects}

This study analyzed the KNHANES VII-1, 2016. The KNHANES has been examined since 1998 by Korea Centers for Disease Control and Prevention (CDC) to assess the health status of the Korean. This survey was the probability-cluster, stratified and multistage survey of a representative sample of the noninstitutionalized population in order to assess the health status in the Korean population. Annually, 4,600 households, 10,000-12,000 individuals are selected from a panel.

The KNHANES VII-1 was a cross-sectional and nationally representative survey consisting of the nutrition survey, the health interview, and the health examination. Among a total of 8,150 persons aged 19 and older who completed KNHANES VII-1, we used data of 1,244 whose age was 65 years and older and whose HGS, body mass index (BMI), and MetS data (Fig. 1).

8,150 KNHANES VII-1, 2016

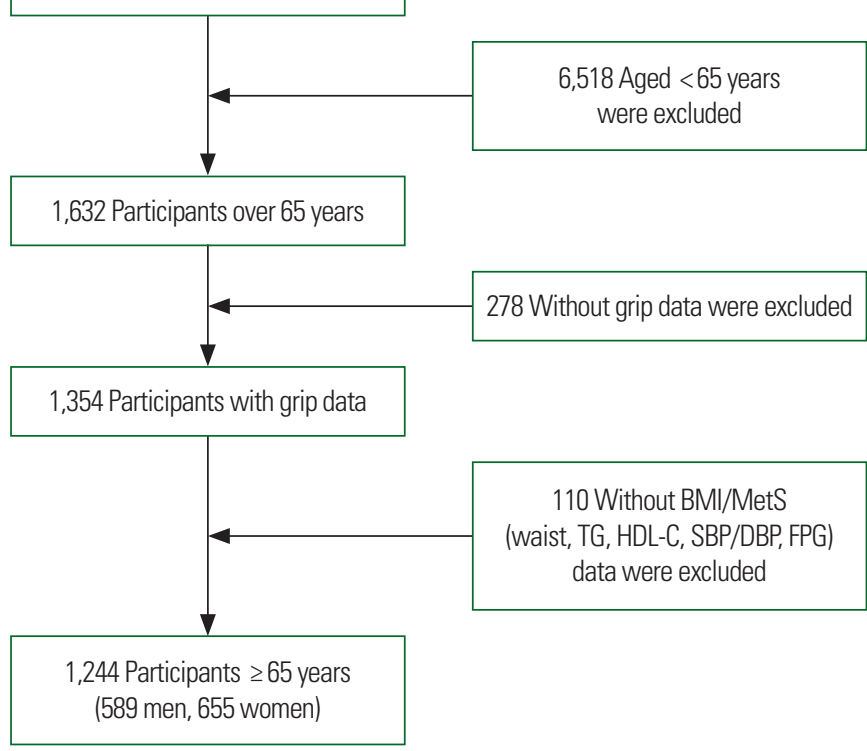

Figure 1. Flow diagram for selection of study subjects. KNHANES, Korea National Health and Nutrition Examination Survey; BMI, body mass index; MetS, metabolic syndrome; TG, triglyceride; HDL-C, high-density lipoprotein; SBP, systolic blood pressure; $\mathrm{DBP}$, diastolic blood pressure; $\mathrm{FPG}$, fasting plasma glucose. 


\section{Ethics statement and data access}

Access to the KNHANES data was acquired after receiving approval by the Institutional Review Board (IRB) of the Korea CDC (2013-12EXP-03-5C). This study is a retrospective study that used and analyzed the data from the 2016 KNHANES survey; therefore, approval from IRB was not required. Because the dataset did not include personal information and participant gave the consent to the KNHANES, further ethical approval for the use of open KNHANES data was exempted from the IRB.

\section{Data collection}

The health examinations included medical history taking, a questionnaire on health-related behaviors, physical examination, anthropometric and biochemical measurements. Physical examinations were performed by trained medical staff following standardized procedures. In demographic variables, family income was categorized into quartiles as Q1 ( $<25$ th percentile), Q2 (25th-49th percentile), Q3 (50th-74th percentile), and Q4 ( $\geq 75$ th percentile). Further, education was classified as below elementary school, middle school graduate, high school graduate, and college graduate or above.

Participants were asked about health-related behaviors, including cigarette smoking status and regular exercise. Smoking status was indicated as "never," "former," and "current" based on their smoking status at the time of the survey. Regular aerobic exercise was indicated as "yes" when the subject participated moderate or strenuous exercise regularly more than 2 hours and 30 minutes per week for moderate exercise that causes slightly increased respiration and heart rate; more than 1 hour and 15 minutes for vigorous exercise. Regular resistance exercise was indicated as "yes" when the subjects participated in resistance exercises such as push-up, arm-curls, and sit-up at least 2 days per week.

Waist circumference (WC) was measured at a middle point between the last rib and the iliac crest. Systolic blood pressure (SBP) and diastolic blood pressure (DBP) measured in the sitting position and reading were recorded twice at 5-minute intervals and averaged for analysis. All the measurement information of blood samples including FPG level, total cholesterol (TC), TG level, HDL-C levels, glycosylated hemoglobin (HbAlc) level were reported in previously shown in KNHANES studies. ${ }^{19}$

\section{Assessment of HGS and MetS parameters}

HGS was measured in each hand three times with a digital grip strength dynamometer (TKK 5401; Takei, Tokyo, Japan). Trained staff instructed the standing participants to hold the dynamometer with the distal interphalangeal finger joints of the hand at $90^{\circ}$ to the handle and to squeeze the handle as hard as they could. A resting interval of 30 seconds was allowed between each measurement. A measure of three times was reported as an average.

Recently, using BMI to adjust for HGS has been recommended as a muscle quality index. ${ }^{20}$ Thus, we used RHGS instead of absolute HGS, defined as the average value for maximum grip strength of the dominant hand divided by BMI which was calculated as weight divided by height squared $\left(\mathrm{kg} / \mathrm{m}^{2}\right)$.

The definition of the MetS proposed by the US National Cholesterol Education Program Adult Treatment Panel $\mathrm{III}^{21}$ was applied for this study. Participants with three or more of the following five criteria were defined as having the MetS: high blood pressure (SBP/ DBP over 130 and $\geq 85 \mathrm{mmHg}$ ) or the use of antihypertensive medication, elevated fasting blood glucose $(\geq 110 \mathrm{mg} / \mathrm{dL})$ or using the antihyperglycemic medication, TG ( $\geq 150 \mathrm{mg} / \mathrm{dL}$ ), low HDL-C (men, $<40 \mathrm{mg} / \mathrm{dL}$; women, $<50 \mathrm{mg} / \mathrm{dL}$ ), and central obesity, as measured by a WC of $\geq 85 \mathrm{~cm}$ for women and $\geq 90 \mathrm{~cm}$ for men.

\section{Statistical analyses}

The complex sample analysis was conducted for the KNHANES data for weighing all values following the guideline given at the workshop by the Korea CDC. Due to the significant sex differences in RHGS data, data of men and women were separated in this study. Sex difference in demographic and anthropometric characteristics was compared using the independent t-test or chi-square test. RHGS was divided into the quartiles. Multivariate logistic regression analysis was analyzed to investigate the association between the quartiles of RHGS and MetS itself or its parameters (SBP, DBP, FPG, HbA1C, TC, TG, HDL-C, and WC).

Statistical analysis was conducted with the IBM SPSS version 23.0 (IBM Corp., Armonk, NY, USA). Continuous and categorical variables were expressed as mean \pm standard error $(\mathrm{SE})$ and number (\%), respectively. The $P$-values less than 0.05 were considered as statistically significant. 
Table 1. General characteristics of the study population by sex

\begin{tabular}{|c|c|c|c|c|c|}
\hline Variable & Classification & Total $(n=1,244)$ & Male $(n=589)$ & Female $(\mathrm{n}=655)$ & tor $\chi^{2}(P)$ \\
\hline Age (yr) & & $72.56 \pm 0.14$ & $72.29 \pm 0.20$ & $72.79 \pm 0.20$ & $-1.763^{*}(0.078)$ \\
\hline \multirow[t]{4}{*}{ Education $(n=1,187)$} & Elementary school or below & $688(58.0)$ & $233(41.3)$ & $455(73.0)$ & $143.223^{\dagger}(<0.001)$ \\
\hline & Middle school & $182(15.3)$ & $97(17.2)$ & $85(13.6)$ & \\
\hline & High school & $190(16.0)$ & $135(23.9)$ & $55(8.8)$ & \\
\hline & College or above & $127(10.7)$ & $99(17.6)$ & $28(4.5)$ & \\
\hline \multirow[t]{4}{*}{ Household income $^{\ddagger}(n=1,233)$} & 01 & $588(45.8)$ & $241(41.5)$ & $347(53.2)$ & $18.687^{\dagger}(<0.001)$ \\
\hline & 02 & $388(30.2)$ & $171(29.4)$ & $167(25.6)$ & \\
\hline & 03 & $182(14.2)$ & $103(17.7)$ & $79(12.1)$ & \\
\hline & 04 & $125(9.7)$ & $66(11.4)$ & $59(9.0)$ & \\
\hline \multirow[t]{3}{*}{ Smoking status $(n=1,216)$} & Never & $724(59.5)$ & $130(22.5)$ & $594(93.2)$ & $632.626^{\dagger}(<0.001)$ \\
\hline & Former & $376(30.9)$ & $349(60.3)$ & $27(4.2)$ & \\
\hline & Current & $116(9.5)$ & $100(17.3)$ & $16(2.5)$ & \\
\hline \multirow[t]{2}{*}{ Regular aerobic exercise $(n=1,186)$} & No & $768(64.8)$ & $321(56.9)$ & $447(71.9)$ & $28.967^{\dagger}(<0.001)$ \\
\hline & Yes & $418(35.2)$ & $243(43.1)$ & $175(28.1)$ & \\
\hline \multirow[t]{2}{*}{ Regular resistance exercise $(n=1,191)$} & No & $973(81.7)$ & $414(73.3)$ & $559(89.3)$ & $50.985^{\dagger}(<0.001)$ \\
\hline & Yes & $218(18.3)$ & $151(26.7)$ & $67(10.7)$ & \\
\hline \multirow[t]{3}{*}{$\operatorname{BMI}(n=1,244)$} & Underweight & $32(2.6)$ & $18(3.1)$ & $14(2.1)$ & $15.555^{\dagger}(<0.001)$ \\
\hline & Normal weight & $738(59.3)$ & $380(64.5)$ & $358(54.7)$ & \\
\hline & Overweight/obese & $474(38.1)$ & $191(32.4)$ & $283(43.2)$ & \\
\hline MetS $(n=1,244)$ & Non-MetS & $554(44.5)$ & $322(54.7)$ & $232(35.4)$ & $46.520^{\dagger}(<0.001)$ \\
\hline
\end{tabular}

Values are presented as mean \pm standard error or number (\%).

${ }^{*}$ t-test; ${ }^{\dagger}$ Chi-square test; ${ }^{\ddagger} 01$ (< 25th percentile), 02 (25th- 49th percentile), 03 (50th-74th percentile), and 04 ( $\geq 75$ th percentile).

$\mathrm{BMI}$, body mass index; MetS, metabolic syndrome.

\section{RESULTS}

The baseline general and clinical characteristics of subjects by gender $(n=1,244)$ are shown in Table 1 . The study population included 589 men $(47.35 \%)$ and 655 women (52.65\%) aged 65 and older from the KNHANES VII-1. The average age of the study population was 72.56 years (SE, 0.14; range, $65-80$ years; men, 72.29 years; women, 72.79 years). There was no significant difference in mean age between men and women. However, education, household income, smoking status, regular exercise, BMI and prevalence of MetS differed significantly between men and women (Table 1).

The cardiovascular risk factors of weighted samples by sex are shown in Table 2. Weight, SBP, WC, levels of FPG, TC, HDL-C, absolute HGS, and RHGS differed significantly between men and women. In men, weight, WC, FPG, absolute HGS, and RHGS were significantly higher than women, but SBP, TC, HDL-C of men were significantly lower than women. The mean of absolute right HGS was $26.94 \mathrm{~kg}$ (men, $34.26 \mathrm{~kg}$; women, $20.36 \mathrm{~kg}$; $P<0.001$ ), and the mean of RHGS was 1.13 (men, 1.45; women,
Table 2. Cardiovascular risk factors of weighted samples by sex

\begin{tabular}{lrrl}
\hline Variable & Male $(\mathrm{n}=589)$ & Female $(\mathrm{n}=655)$ & $\mathrm{t}(P)^{*}$ \\
\hline Weight $(\mathrm{kg})$ & $65.26 \pm 0.38$ & $57.09 \pm 0.34$ & $15.962(<0.001)$ \\
$\mathrm{SBP}(\mathrm{mmHg})$ & $126.41 \pm 0.66$ & $130.34 \pm 0.68$ & $-4.109(<0.001)$ \\
$\mathrm{DBP}(\mathrm{mmHg})$ & $72.19 \pm 0.41$ & $72.99 \pm 0.38$ & $-1.418(0.157)$ \\
$\mathrm{BMI}\left(\mathrm{kg} / \mathrm{m}^{2}\right)$ & $23.79 \pm 0.12$ & $24.71 \pm 0.13$ & $-5.081(<0.001)$ \\
WC $(\mathrm{cm})$ & $87.10 \pm 0.35$ & $85.45 \pm 0.36$ & $3.294(0.001)$ \\
FPG (mg/dL) & $111.72 \pm 1.31$ & $107.15 \pm 1.01$ & $2.779(0.006)$ \\
HbA1C $(\%)$ & $6.07 \pm 0.04$ & $6.03 \pm 0.32$ & $0.615(0.538)$ \\
TC $(\mathrm{mg} / \mathrm{dL})$ & $179.94 \pm 1.52$ & $190.75 \pm 1.54$ & $-4.992(<0.001)$ \\
TG (mg/dL) & $140.83 \pm 3.74$ & $141.88 \pm 3.42$ & $-0.211(0.833)$ \\
HDL-C (mg/dL) & $45.85 \pm 0.48$ & $49.36 \pm 0.47$ & $-5.187(<0.001)$ \\
LDL-C (mg/dL) & $107.89 \pm 3.72$ & $112.02 \pm 3.31$ & $-0.831(0.407)$ \\
Absolute right HGS (kg) & $34.26 \pm 0.29$ & $20.36 \pm 0.19$ & $40.050(<0.001)$ \\
RHGS (kg/BMI) & $1.45 \pm 0.01$ & $0.84 \pm 0.01$ & $40.379(<0.001)$ \\
\hline
\end{tabular}

Values are presented as mean \pm standard error.

${ }^{*}$ t-test.

SBP, systolic blood pressure; DBP, diastolic blood pressure; BMI, body mass index; WC, waist circumference; FPG, fasting plasma glucose; $\mathrm{HbA1C}$, glycosylated hemoglobin; TC, total cholesterol; TG, triglyceride; HDL-C, high-density lipoprotein cholesterol; LDLC, low-density lipoprotein cholesterol; HGS, handgrip strength; RHGS, relative handgrip strength. 
0.84) and there was a significant sex difference in both absolute HGS and RHGS. There were no sex differences regarding DBP, HbAlc, TG, and LDL-C (Table 2).

The association between RHGS and components of the MetS are shown in Table 3. WC was negatively associated with RHGS in both men and women $(\mathrm{r}=-0.338, \mathrm{r}=-0.396 ; P<0.001$, respectively). Weight and $\mathrm{HbA1C}$ are negatively associated with RHGS in both sexes, but HDL-C was positively partial correlated with RHGS in both sex. Aerobic exercise is partial positive correlated with RHGS in both sexes $(P<0.001, P<0.038$, respectively), conversely, strength exercise was positively correlated with RHGS in men only $(P<0.001)$ (Table 3$)$.

Table 3. Relationship between relative handgrip strength and metabolic parameters including components of metabolic syndrome

\begin{tabular}{|c|c|c|}
\hline \multirow{2}{*}{ Variable } & \multicolumn{2}{|c|}{ Partial $r^{*}(P)$} \\
\hline & Male $(n=589)$ & Female $(n=655)$ \\
\hline WC (cm) & $-0.338(<0.001)$ & $-0.396(<0.001)$ \\
\hline $\mathrm{SBP}(\mathrm{mmHg})$ & $0.059(0.451)$ & $0.015(0.705)$ \\
\hline Weight (kg) & $-0.193(<0.001)$ & $-0.298(<0.001)$ \\
\hline $\mathrm{FPG}(\mathrm{mg} / \mathrm{dL})$ & $-0.117(0.004)$ & $-0.068(0.092)$ \\
\hline $\mathrm{HbA1C}(\%)$ & $-0.126(0.002)$ & $-0.080(0.041)$ \\
\hline $\mathrm{TC}(\mathrm{mg} / \mathrm{dL})$ & $-0.106(0.011)$ & $0.040(0.314)$ \\
\hline $\mathrm{TG}(\mathrm{mg} / \mathrm{dL})$ & $-0.073(0.079)$ & $-0.123(0.002)$ \\
\hline $\mathrm{HDL}-\mathrm{C}(\mathrm{mg} / \mathrm{dL})$ & $0.229(0.011)$ & $0.124(0.002)$ \\
\hline Smoking & $0.034(0.420)$ & $0.151(<0.001)$ \\
\hline Aerobic exercise & $0.154(<0.001)$ & $0.083(<0.038)$ \\
\hline Strength exercise & $0.201(<0.001)$ & $0.056(0.164)$ \\
\hline
\end{tabular}

*Pearson's correlation coefficient adjusted for age.

WC, waist circumference; SBP, systolic blood pressure; FPG, fasting plasma glucose; $\mathrm{HbA1C}$, glycosylated hemoglobin; TC, total cholesterol; TG, triglyceride; HDL-C, highdensity lipoprotein cholesterol.
Table 4 shows the results of a logistic regression analysis using MetS as an objective variable and confounding factors including age, education, family income, marital status, and regular exercise participation (aerobic exercise \& resistance exercise) as explanatory variables were performed with subjects categorized by sex. Of the subjects, 267 men (45.3\%) and 423 women (64.6\%) had MetS. The prevalence of MetS significantly decreased in relation to increased RHGS in both men and women $(\mathrm{F}=35.16, P<0.001$ vs. $\mathrm{F}=31.78$, $P<0.001$, respectively). The multivariate-adjusted odds ratio $(95 \%$ $\mathrm{CI}$ ) in the lowest quartile compared with the highest for MetS was 1.00, 0.74 (0.46-1.21), 0.93 (0.57-1.54), 0.23 (0.13-0.40) in men and 1.00, 0.73 (0.44-1.21), 0.52(0.31-0.85), $0.28(0.17-0.47)$ in women for Q1, Q2, Q3, and Q4, respectively (Tables 4 and 5).

\section{DISCUSSION}

The current study examined the association between MetS and

Table 4. Association between relative handgrip strength and prevalence of MetS

\begin{tabular}{lrrrrr}
\hline \multirow{2}{*}{ Characteristics } & \multicolumn{4}{c}{ Relative handgrip strength (kg/BMI) } & \multirow{2}{*}{$F(P)$} \\
\cline { 2 - 5 } & Quartile 1 & Quartile 2 & Quartile 3 & Quartile 4 & \\
\hline Male $(n=589)^{*}$ & & & & \multicolumn{3}{c}{$35.16(<0.001)$} \\
Non-MetS & $69(21.4)$ & $75(23.3)$ & $67(20.8)$ & $111(34.5)$ & \\
MetS & $78(29.2)$ & $73(27.3)$ & $80(30.0)$ & $36(13.5)$ & \\
Female $(n=655)^{\dagger}$ & & & & & $31.78(<0.001)$ \\
Non-MetS & $37(15.9)$ & $50(21.6)$ & $61(26.3)$ & $84(36.2)$ & \\
MetS & $127(30.0)$ & $114(27.0)$ & $102(24.0)$ & $80(18.9)$ & \\
\hline
\end{tabular}

Values are presented as number (\%).

*Male: quartile $1(n=147)$, quartile 2 ( $n=148)$, quartile $3(n=147)$, quartile $4(n=147)$; 'Female: quartile $1(n=164)$, quartile $2(n=164)$, quartile $3(n=163)$, quartile $4(n=164)$. MetS, metabolic syndrome; BMl, body mass index.

Table 5. Odds ratio and 95\% $\mathrm{Cl}$ for the metabolic syndrome of subjects according to quartile of relative handgrip strength

\begin{tabular}{|c|c|c|c|c|c|}
\hline Variable & Quartile 1 & Quartile 2 & Quartile 3 & Quartile 4 & $P$ \\
\hline \multicolumn{6}{|l|}{$\operatorname{Male}^{*}(n=589)$} \\
\hline Unadjusted & 1 & $0.91(0.57-1.45)$ & $0.93(0.58-1.47)$ & $0.25(0.14-0.44)$ & $<0.001$ \\
\hline Model 1 & 1 & $0.73(0.45-1.16)$ & $0.83(0.51-1.35)$ & $0.20(0.12-0.39)$ & 0.024 \\
\hline Model 2 & 1 & $0.74(0.46-1.21)$ & $0.93(0.57-1.54)$ & $0.23(0.13-0.40)$ & $<0.001$ \\
\hline \multicolumn{6}{|c|}{ Female $^{\dagger}(n=655)$} \\
\hline Unadjusted & 1 & $0.66(0.41-1.09)$ & $0.49(0.30-0.79)$ & $0.28(0.17-0.45)$ & $<0.001$ \\
\hline Model 1 & 1 & $0.61(0.37-0.99)$ & $0.48(0.30-0.79)$ & $0.25(0.15-0.41)$ & $<0.001$ \\
\hline Model 2 & 1 & $0.73(0.44-1.21)$ & $0.52(0.31-0.85)$ & $0.28(0.17-0.47)$ & $<0.001$ \\
\hline
\end{tabular}

Values are presented as odds ratio (95\% CI). Model 1: adjusted for age, Model 2: adjusted for age, smoking status, education, family income, drinking, aerobic exercise, resistance exercise.

*Male: quartile 1 ( $n=147)$, quartile 2 ( $n=148)$, quartile $3(n=147)$, quartile 4 ( $n=147)$; ${ }^{\dagger}$ Female: quartile 1 ( $\left.n=164\right)$, quartile 2 ( $\left.n=164\right)$, quartile 3 ( $\left.n=163\right)$, quartile 4 ( $\left.n=164\right)$. $\mathrm{Cl}$, confidence interval. 
RHGS in the elderly of a nationally representative sample of Korea, with two key findings. First, the current study found significant associations between higher RHGS and more favorable features of MetS including body weight, FPG, total cholesterol, HDL-cholesterol in men and WC, body weight, and TG in women. These findings are in line with previous physiologic research in older adults. ${ }^{16,22-24}$ However, these findings are inconsistent with studies that include younger adults aged over 20 years and showed counterintuitive associations of grip strength with poor SBP, TG. ${ }^{24}$

One explanation for contradictory findings among previous studies may be the use of absolute versus RHGS. Some features of MetS may be more strongly associated with an absolute measure. The use of RHGS, which was calculated absolute HGS divided by BMI would aim to examine the independent contribution of body composition and strength. Since cardiometabolic health and aging are affected by sarcopenia, it would be more appropriate to use RHGS in the matter of MetS in senior with the consideration of aging body composition.

In the current study, we also found sex differences in the association between RHGS and TC, TG, and FPG. Consistent with a previous study ${ }^{4}$, we observed that RHGS was associated with a lower risk of impaired fasting glucose level in men alone. This may be related to the level of testosterone which is linked to both lipid, fasting glucose level and muscle tissue. It has been proposed that high glucose level has a direct adverse effect on muscle contraction and force generation. At the cellular level, different status of muscle aging by different hormonal level may induce a reduction in both the number of myocytes in different by sex. This induces adverse metabolic consequences in terms of reduced glucose uptake, because of sex difference in muscle mass. ${ }^{25}$

Second, the current study found a significant association between RHGS and MetS after controlling for the covariates including age, smoking status, education, family income, drinking, aerobic exercise, and resistance exercise. That is, lower prevalence of MetS was found in the highest quartile RHGS groups compared to the lowest quartile RHGS group in both sexes. These findings may imply that poorer RHGS indicates a higher risk of MetS as well as high risk of cardiovascular biomarkers. These findings are in line with those of several previous studies. ${ }^{14,23}$

Consequently, monitoring RHGS may be critical to identify the risk of MetS in older adults in Korea. Considering that in terms of prevalence of MetS, CVDs has ranked the second and third for mortality in Korean older population over 65 years of age ${ }^{26}$, this finding would be significant in demonstrating RHGS as a potential tool for identifying MetS.

Numerous studies have shown that physical function in seniors is associated with health-related issues including mortality and exacerbation with the current disease. ${ }^{27}$ Despite its importance, physical function is rarely measured in a clinical setting or practice because of a certain amount of time and space as well as a safety issue. Besides, it is also essential to understand the factors affecting the disabling process and the sequence of health events when the public health professional target the preservation of physical function for the aging population. Therefore, the attempt to find out a simple way of measuring physical function and apply proper intervention in time in the elderly should be critical. In this perspective, the RHGS is one of the simple and safe ways of the proposed alternatives. Our study highlights the importance of assessing RHGS from the perspective of MetS.

The current study includes a couple of limitations. First, due to the population-based cross-sectional study, it is impossible to ascertain the direction of the association between muscle strength and metabolic function. In other words, older persons with MetS may have poorer diets and reduced further physical activity which result in low HGS. Further, we could not assess longitudinal changes in RHGS or the association between RHGS and exacerbation of MetS. Thus, RCT interventional or longitudinal studies are required to investigate this further.

Second, because of the data availability, it was not possible to examine the relationship between RHGS and insulin resistance. This has the potential for adverse metabolic consequences in terms of reduced glucose uptake and hyperglycemia. It has been suggested that hyperglycemia has an adverse effect on muscle contractile function. ${ }^{25}$

Despite the above limitations, in our study, the sample was representative of the population using the national registration lists. Thus it is significant findings that the low relative grip strength was independently associated with MetS in the older population. In the present cohort, participants with MetS were more likely to be women. Older Korean women were more likely to be classified as 
less educated, sedentary, poor and more overweight or obese than older men. A better understanding of these sociodemographic and behavioral characteristics could potentially help to develop more accurate recognition strategies and effective intervention leading to reduced frailty. Therefore this group of older adults should be given more focus in terms of exercise program which enhance grip strength.

In conclusion, RHGS was significantly associated with MetS in Korean older population. Additional longitudinal and interventional studies are required to develop strategies in this population.

\section{CONFLICTS OF INTEREST}

The author declares no conflict of interest.

\section{ACKNOWLEDGMENTS}

This work was supported by Kangnam University Research grant.

\section{REFERENCES}

1. Grundy SM, Cleeman JI, Daniels SR, Donato KA, Eckel RH, Franklin BA, et al. Diagnosis and management of the metabolic syndrome: an American Heart Association/National Heart, Lung, and Blood Institute Scientific Statement. Circulation 2005;112:2735-52.

2. Al-Rubeaan K, Bawazeer N, Al Farsi Y, Youssef AM, Al-Yahya AA, AlQumaidi $\mathrm{H}$, et al. Prevalence of metabolic syndrome in Saudi Arabia: a cross sectional study. BMC Endocr Disord 2018;18:16.

3. de Paula JA, Moreira OC, da Silva CD, Silva DS, dos Santos Amorim PR. Metabolic syndrome prevalence in elderly of urban and rural communities participants in the HIPERDIA in the city of Coimbra/MG, Brazil. Invest Educ Enferm 2015;33: 325-33.

4. Li R, Li W, Lun Z, Zhang H, Sun Z, Kanu JS, et al. Prevalence of metabolic syndrome in Mainland China: a meta-analysis of published studies. BMC Public Health 2016;16:296.

5. Lau DC, Yan H, Dhillon B. Metabolic syndrome: a marker of patients at high cardiovascular risk. Can J Cardiol 2006;22
Suppl B:85B-90B.

6. Lim S, Kim JH, Yoon JW, Kang SM, Choi SH, Park YJ, et al. Sarcopenic obesity: prevalence and association with metabolic syndrome in the Korean Longitudinal Study on Health and Aging (KLoSHA). Diabetes Care 2010;33:1652-4.

7. Wu SH, Liu Z, Ho SC. Metabolic syndrome and all-cause mortality: a meta-analysis of prospective cohort studies. Eur J Epidemiol 2010;25:375-84.

8. Ford ES. Risks for all-cause mortality, cardiovascular disease, and diabetes associated with the metabolic syndrome: a summary of the evidence. Diabetes Care 2005;28:1769-78.

9. Chang TT, Yen YC. Metabolic syndrome predicts cognitive decline in community-dwelling elderly people: a 10-year cohort study. Neuropsychiatry (London) 2018;8:96-101.

10. Cruz-Jentoft AJ, Bahat G, Bauer J, Boirie Y, Bruyère O, Cederholm T, et al. Sarcopenia: revised European consensus on definition and diagnosis. Age Ageing 2019;48:16-31.

11. Wind AE, Takken T, Helders PJ, Engelbert RH. Is grip strength a predictor for total muscle strength in healthy children, adolescents, and young adults? Eur J Pediatr 2010;169:281-7.

12. Batsis JA, Mackenzie TA, Barre LK, Lopez-Jimenez F, Bartels SJ. Sarcopenia, sarcopenic obesity and mortality in older adults: results from the National Health and Nutrition Examination Survey III. Eur J Clin Nutr 2014;68:1001-7.

13. Günther CM, Bürger A, Rickert M, Crispin A, Schulz CU. Grip strength in healthy caucasian adults: reference values. J Hand Surg Am 2008;33:558-65.

14. Bohannon RW. Muscle strength: clinical and prognostic value of hand-grip dynamometry. Curr Opin Clin Nutr Metab Care 2015;18:465-70.

15. Wu Y, Wang W, Liu T, Zhang D. Association of grip strength with risk of all-cause mortality, cardiovascular diseases, and cancer in community-dwelling populations: a meta-analysis of prospective cohort studies. J Am Med Dir Assoc 2017;18:551. e17-551.e35.

16. Gubelmann C, Vollenweider P, Marques-Vidal P. No association between grip strength and cardiovascular risk: the CoLaus population-based study. Int J Cardiol 2017;236:478-82.

17. Lee WJ, Peng LN, Chiou ST, Chen LK. Relative handgrip strength is a simple indicator of cardiometabolic risk among 
middle-aged and older people: a nationwide population-based study in Taiwan. PLoS One 2016;11:e0160876.

18. Yates T, Zaccardi F, Dhalwani NN, Davies MJ, Bakrania K, Celis-Morales CA, et al. Association of walking pace and handgrip strength with all-cause, cardiovascular, and cancer mortality: a UK Biobank observational study. Eur Heart J 2017;38: 3232-40.

19. Studenski SA, Peters KW, Alley DE, Cawthon PM, McLean RR, Harris TB, et al. The FNIH sarcopenia project: rationale, study description, conference recommendations, and final estimates. J Gerontol A Biol Sci Med Sci 2014;69:547-58.

20. Lee MR, Jung SM, Kim HS, Kim YB. Association of muscle strength with cardiovascular risk in Korean adults: findings from the Korea National Health and Nutrition Examination Survey (KNHANES) VI to VII (2014-2016). Medicine (Baltimore) 2018;97:e13240.

21. Expert Panel on Detection, Evaluation, and Treatment of High Blood Cholesterol in Adults. Executive summary of the third report of the National Cholesterol Education Program (NCEP) expert panel on detection, evaluation, and treatment of high blood cholesterol in adults (Adult Treatment Panel III). JAMA 2001;285:2486-97.
22. Sayer AA, Syddall HE, Martin HJ, Dennison EM, Roberts HC, Cooper C. Is grip strength associated with health-related quality of life? Findings from the Hertfordshire Cohort Study. Age Ageing 2006;35:409-15.

23. Stenholm S, Sallinen J, Koster A, Rantanen T, Sainio P, Heliövaara $\mathrm{M}$, et al. Association between obesity history and hand grip strength in older adults: exploring the roles of inflammation and insulin resistance as mediating factors. J Gerontol A Biol Sci Med Sci 2011;66:341-8.

24. Lawman HG, Troiano RP, Perna FM, Wang CY, Fryar CD, Ogden CL. Associations of relative handgrip strength and cardiovascular disease biomarkers in U.S. Adults, 2011-2012. Am J Prev Med 2016;50:677-83.

25. Helander I, Westerblad H, Katz A. Effects of glucose on contractile function, $[\mathrm{Ca} 2+] \mathrm{i}$, and glycogen in isolated mouse skeletal muscle. Am J Physiol Cell Physiol 2002;282:C1306-12.

26. Statistics Korea. Elderly statistics in Korea. Daejeon: Statistics Korea; 2018.

27. Lee SH, Lee Y, Seo JH, Kim YA. Association between exercise and metabolic syndrome in Koreans. J Obes Metab Syndr 2018; 27:117-24. 